\title{
Formation and the effects of the thermo-residual stress on mechanical properties of the thermoplastic composites
}

\author{
Wang Changchun ${ }^{1, a^{*}}$, Yue Guangquan ${ }^{1, b}$ Zhang Jiazhen $^{1, c}$, Liu Jianguang ${ }^{1, d}$, \\ and Li $\mathrm{Jin}^{2, \mathrm{e}}$ \\ ${ }^{1}$ Beijing Aeronautical Science and Technology Research Institute, Commercial Aircraft \\ Corporation of China Ltd., Beijing 102211, China; \\ ${ }^{2}$ Key Laboratory of Ningxia for Photovoltaic Materials, Ningxia University, Yinchuan 750021 , \\ China \\ ªwangchch2007@163.com, byueguangquan@comac.cc, 'chhangjiazhen@comac.cc, \\ diujianguang@comac.cc, ${ }^{\mathrm{I}} \mathrm{li}$-jin@163.com
}

Keywords: thermoplastic composites; thermo-residual stress; formation; part properties

Abstract. The thermoplastic composites have widely regarded as high property structural materials for aerospace field for the excellent advantages, which were usually procured based on the moulding process under the high processing temperature and cooling rate. The thermo-residual stresses of the composites will be produced for the mismatch in thermal expansion coefficients between the fiber and matrix, as well as the anisotropy property of the adjacent layers of the plate and temperature gradient of the laminates structures having a certain thickness. The thermo-residual stresses play an important role on the mechanical properties of the obtained thermoplastic composites, which should be taken into consideration in the designing process. It will good to study the formation mechanism and the effects of thermo-residual stresses on the mechanical properties of thermoplastic composites to better utility and control its residual stress level. In present paper, the formation reasons of the thermo-residual stresses were firstly studies and analyzed from three different levels, the methods for controlling and decreasing the thermo-residual stresses were also discussed. In addition, the effects of the residual stresses on the thermoplastic composites properties were also studied from the aspects of dominating by the reinforcement and the polymer matrix.

\section{Introduction}

Fiber reinforced thermoplastic composites have been widely used in the aerospace for the high toughness, long storage period, and short molding cycle, which was regarded as the promising advanced aeronautical structure materials [1,2]. The mechanical properties of the thermoplastic composites will be influenced by the crystallinity of the polymer matrix, which will be affected by the thermo-residual stresses in the obtained composites. Thermo-residual stresses may be formed during the processes of the thermoplastic composites when they were cooling down from the high process temperature to the service temperature for the mismatch of the thermal expansion coefficient between fiber and polymer matrix, as well as the anisotropy property of the adjacent layers of the laminates and the possible temperature gradient distribution of the studied laminates, which must be taken into consideration during the designing process of the composite components [3].

The there is the hot research topic about the thermo-residual stresses for the widely appilication. In present study, the formation causes and the development of thermo-residual stresses was firstly introduced from three different levels, in which the method of controlling and decreasing the residual stresses was also discussed. In addition, the effects of the thermo-residual stresses on the obtained thermoplastic composites were followed studied as well.

\section{Formation mechanism of thermo-residual stresses}

Formation causes. The fromation of thermo-residual stresses in the molding fiber reinforced polyether sulfone (PES) was firstly studied by Nairn and Zoller et al. by the photo-elastic method at 1985 [4]. 
Favre tested the residual stresses of the composites during the producing process with the same method; moreover, the effects of temperature and time on residual stresses were also studied in their experiments [5]. The diagram of the formation of thermo-residual stresses between the fiber and polymer matrix in the cooling down process is shown in the Fig. 1, which can see that there is good match between each other at the high process temperature for the thermo-residual can be released in this stage. However, the mismatch of the deformation will be occured as they endure the high cooling rate to the service temperature, therefore the thermo-residual stress will be 'fixed" into the final composites. The formation causes of the thermo-residual stresses can be divided into three different levels, i.e. constituent materials level, laminate plates level and structure parts level, the specific classification of thermo-residual stresses is given in table 1.

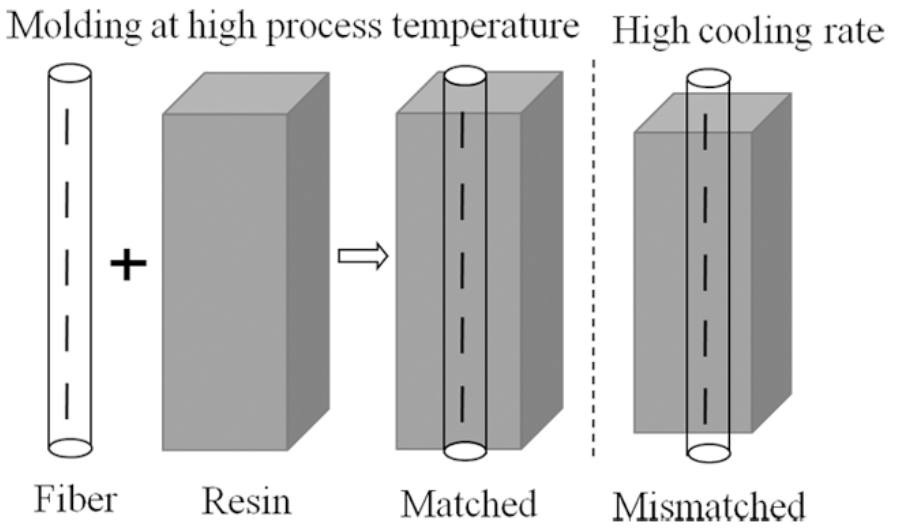

Fig. 1 Diagram of the interaction between the fiber and matrix of the thermoplastic composites Table 1 Classification of three level of the thermo-residual stresses of the thermoplastic composites

Classification levels Interaction mechanism of the thermo-residual stresses

Constitute materials

Laminate plates

Structure parts
Deformation mismatch of the reinforcement fiber and polymer matrix in the cooling process, the matrix and fiber of the obtained composites will be applied by the stretch and compression action, respectively. Mismatch of the thermal expansion coefficient of the adjacent layer along the radial and transverse direction of the thermoplastic composites, as well as the transverse crystalline effect Temperature gradient formed during the cooling process as the thermoplastic composites having a certain thickness

\section{Constituents level thermo-residual stresses}

Influence of the fiber on the thermo-residual stresses. In the aerosplace field, the mostly widely used reinforment material is carbon fiber yarn and its fabric, the difference in the thermal expansion coefficient of the fiber along and vertical will cause the thermo-residual stresses during the cooling process and then lead to the fiber deformation [6]. However, the deformation caused by the fiber is much smaller than that caused by the polymer matrix due to the thermal expansion coefficient of the fiber is much smaller than the polymer matrix deformation in the whole process of thermoplastic composite materials according to the previous studies, which plays the tiny influence on the thermo-residual stresses in the thermoplastic composites caused by the fiber deformation and can be neglected.

Influence of the matrix on the thermo-residual stresses. The thermoplastic matrix presents the viscoelastic properties in the cooling process from the high temperature to the service temperature, in which the thermo-residual stresses will be released for the molecular chain has enough energy. As the temperature continue to reduce, the matrix has the thermal elasticity properties during this stage and the thermo-residual stresses at this stage would be 'fixed' in the composites. The cooling rate has the important role on the residual stresses; higher cooling rate will be lower the crystallinity levels [8]. 
Moreover a higher cooling rate will also compromise relaxation behavior of matrix, and these two causes were competed and interaction on the residual stresses of the thermoplastic composites.

Plate level thermo-residual stresses. In the plate level of the thermo-residual stresses, the influence factors for the constituent materials level will be continue to action in this level; however, the effect of the layup pattern on the thermo-residual stresses during the molding process was major concerned, including the angle-ply, fiber fabric structure and the crystallinity of the polymer matrix. The residual stresses increases with the increasing of the difference between the stress-free temperature (SFT) and service temperature. The SFT can be obtained by heating the obtained thermoplastic composites until the deformation equals zero [9]. The thermo-residual stresses of the thermoplastic composites caused by the temperature gradient of the plate will accumulate.

Structural level thermo-residual stresses. The thermo-residual stresses will be produced when the thickness of the thermoplasric composites having a certain thickness, and it is affected by the cooling process. The residual stresses in the thermoplastic composites maight be different from each other during the cooling process, although with the same crystallinity degree at the start of cooling stage. Annealing method of the composites can be used to relax the thermo-residual stresses and lower the residual stresses gradient, which can improve the crystallinity level of the thermoplastic composites; but it will be significant increase the process time, resulting in an increase in manufacturing costs.

The properties of the mould materials used for the thermoplastic composite also have the important influence on the thermo-residual stresses, which were mainly action from the aspects of thermal and mechanical interaction [10]. The thermal interaction has an important effect on the formation and distribution of global thermo-residual stresses, which is determined by the mould materials properties and the cooling rate on the top and bottom surfaces of the thermoplastic composite. The mechanical interaction is produced by the mismatch of the thermal expansion coefficient between the tool and composite part. It will cause the compressive thermo-residual stresses in the surface plies of the parts near the tool interface and present a through-thickness stress distribution.

\section{Effects of the thermo-residual stresses on the thermoplastic composites performances}

The thermo-residual stresses of the thermoplastic composites produced during the molding process have the important influence on the thermoplastic composites mechanical properties, which will be formed for the thermal expansion coefficient mismatch, layer anisotropy, the interaction between the mold and matrix and cooling rate [11]. The new formed thermo-residual stresses will be compromised the mechanical properties, damage resistance and dimensional stability of the thermoplastic composites. The residual stresses in thermoplastic composites have received widely concerned, in the follows, the effects of which on the mechanical properties of the thermoplastic composites dominated by the fiber and the matrix will be respectively discussed in detailed.

Effects of the thermo-residual stresses on the matrix-dominated properties. The presence of the thermo-residual stresses of the thermoplastic composites will cause the matrix materials under the stretched loading condition, the size of which will be influenced by the fiber volume fraction of the composites. The mechanical properties dominated by the polymer matrix will be severely affected by the thermo-residual stresses, such as moisture absorption property [12]. The compressive residual stresses have the effect of increasing the glass transition temperature; however, the interaction of shear and tensile decrease this effect. The use temperature upper limit of the thermoplastic composites can be improved through designing the layup of the composites to ensure the parts under a needed load cases. D'Amore et al. studied the relationship between the glass transition temperature $\left(T_{\mathrm{g}}\right)$ of the carbon fiber reinforced polyetherimide (PEI) and the pure PEI by using the DSC method, the results show that the modified PEI has a lower $\mathrm{T}_{\mathrm{g}}$ than that of the pure PEI materials [13].

The thermo-residual stresses of the thermoplastic composites will decrease as the temperature difference increase between the test temperature and stress-free temerature with a linear relationship. The deformation of the thermoplastic composites can be reduced when the parts placed in the service temperature due to the stress relaxation effect of the polymer matrix caused by the loading action, 
environmental temperature and humidity, which will all affect the thermo-residual stress level and the final mechanical properties of the composite structures.

The aging effect of matrix material can affect the properties of the thermoplastic composites, the polymer matrix will has the glass properties as the environmental temperature lower than its glass transition temperature, the thermodynamic equilibrium state will change as the change of the elastic modulus of the composites caused the aging effects [14]. The elastic modulus and yield strength of the composite were improved, and the toughness was significantly reduced, which will affect the release of thermo-residual stress. In the oxygen-rich case, the brittleness of the polymer matrix caused by the aging effect will become much seriously than the anaerobic case, and the mechanical properties of which have significantly reduce with the same change trend.

Effects of the interface properties of the fiber and polymer matrix. Unlike with the thermoset composite materials, the bonding interaction between the fiber and the matrix of the thermoplastic composites was the Van der Waals force. The interface shear strength of the obtained composites can be greatly affected by the thermo-residual stresses, which will lead to the debonding damage along the fiber axial and compromise its final mechanical properties [15].

Based on the previous studies, the mechanical properties of the fiber are not serious influenced by the presence of the thermo-residual stresses, however, which will cause the compressive force on the fiber reinforcement and leads to the fiber breakage.

Effects of the thermo-residual stresses on the laminate mechanical properties. For the unidirectional thermoplastic composites, the fiber presents a higher tensile failure strain for the compressive force caused by the thermo-residual stresses, which will also affect the lateral performance of the obtained composites. When the composites are stretched along the fiber axial direction, the strain of the fiber under the compressive interaction present a larger strain compared with the fiber without any force [16]. The tensile strength of the laminate is lower than the unidirectional transverse tensile strength as there are the thermo-residual stresses in the laminates caused by the thermal contraction along the thickness direction.

The compressive performance of the laminate will striking decrease as there are thermo-residual stresses in the parts, what is more important is that the thermo-residual stresses play an important effect on the transverse compressive property as the fiber waviness effect due to the stresses. In the compressive tests, the failure strain of the laminates with thermo-residual stresses decreases, moreover the tensile and compressive modulus are different from each other. The stiffness and interfacial performance of the laminates may be degraded as the presence of the discontinuous of the interlayers caused by the thermo-residual stresses.

The fracture toughness of the composites plays a important role in resistance the crack propagation, which can also affect by the themro-residual stresses for the failure mode I (delamination) and II (layer stratification). The residual stress will significantly reduce the fracture toughness of the composites, especially it can lead to the strikely decrease of the strength of the mode I [17]. Moreover, The thermo-residual stress can seriously affect the fatigue performance of the laminates as leading to the free edge delamination.

\section{The possible defects casused by the thermo-residual stresses}

The reason of the decrease of the mechanical properties caused by the thermo-residual stresses was that the defects maight be formed during the molding process. The follows parts of present study will detailed discuss the effect of the thermo-residual stresses on the aspects of properties of the fiber and the matrix, as well as the interface performance of the fiber and matrix.

The effects of the thermo-residual stresses on the fiber. Unlike with the thermoset composite materials, the bonding interaction between the fiber and the matrix of the thermoplastic composites was the Van der Waals force [18]. The interface shear strength of the obtained composites can be greatly affected by the thermo-residual stresses, which lead to the debonding damage along the fiber axial and compromise its final mechanical properties. 
The effects of the thermo-residual stresses on the fiber and matrix interface properties. The thermo-residual stresses can cause the defects of micro-cracks in the fiber-matrix interface. The crack or delamination will be formed as the thermo-residual stresses was stronger than the interfacial strength, and the residual stresses will release in the matrix as the form of expansion of micro-crack. In the service process, the crack tip will firstly up to yield strength and form a transverse crack which leads to the loss of bearing capacity. The crack density will greatly increase as the thermoplastic composites were endured the thermal cycling, heat treatment, aging and temperature conditions, which will obviously decrease the compressive performance of the obtained composites [19]. Figure 2 shows the typical transverse crack of the surface layer of the carbon fiber/PEI, which seriously reduced the final mechanical properties of the thermoplastic composites.

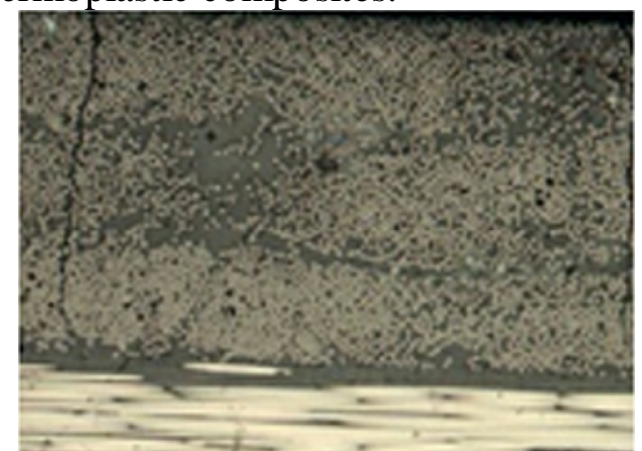

Fig. 2 Transverse crack of the surface layer of the carbon fiber/PEI plate of [03/909/03] $]^{[9]}$

\section{Conclusions}

In present manuscript, the thermo-residual stresses of the thermoplastic composites were discussed and analyzed based on the published publications from three different levels, which were formed by the thermal expansion coefficient of the fiber and polymer matrix, the interaction between the adjacent layers and the temperature uneven distribution of the thermoplastic composites. Moreover, the effects of the residual stresses on the thermoplastic composites were also studied and the methods to control and reduce it was discussed as well, which can be used as the guidance in designing the qualified thermoplastic composites.

\section{Acknowledgements}

The authors would like to acknowledge the financial support of the Beijing Municipal Science and Technology Project for this work, Grant no. Z151100002815021.

\section{References}

[1] J. Bernhardson, R. Shishoo, Effect of parameters on consolidation quality of GF/PP commingled yarn based composites, J. Thermoplast Compos. 13 (2000) 292-313.

[2] G. Schinner, J. Brandt, H. Richer, Recycling carbon-fiber reinforced thermoplastic composites, J. Thermoplast Compos. 9 (1996) 237-245.

[3] J.A. Barnes, G.E. Byerly The formation of residual-stress in laminated thermoplastic composites, Compos. Sci. Technol. 51 (1994) 479-494.

[4] J.A. Nairn, P. Zoller, Matrix solidification and the resulting residual thermal stresses in composites, J. Mater. Sci. 20 (1985) 355-367.

[5] Favre JP. Residual thermal stresses in fiber reinforced composite materials a review[J]. J. Mech. Behav. Mater., 1 (1988) 37-53.

[6] P.P. Parlevliet, H.E.N. Bersee, A. Beukers, Residual stresses in thermoplastic composites-A study of the literature-Part I: Formation of residual stresses, Composites Part A 37 (2006) 1847-1857. 
[7] P.P. Parlevliet, H.E.N. Bersee, A. Beukers, Residual stresses in thermoplastic composites-A study of the literature-Part II: Experimental techniques, Composites Part A 38 (2007) 651-665.

[8] K.S. Kim, H.T. Hahn, R.B. Croman, The effect of cooling rate on residual stresses in a thermoplastic composite, J. Compos. Tech. Res. 11 (1989) 47-52.

[9] P.P. Parlevliet, H.E.N. Bersee, A. Beukers, Residual stresses in thermoplastic composites-A study of the literature-Part III: Effects of thermal residual stresses, Composites Part A 38 (2007) 1581-1596.

[10] S.L. Gao, J.K. Kim, Cooling rate influences in carbon fiber/PEEK composites. Part 1. cystallinity and interface adhesion, Composites Part A, 31 (2000) 517-530.

[11] E.M. Asloun, M. Nardin, J. Schultz, Stress transfer in single-fiber composites: effect of adhesion, elastic modulus of fiber and matrix, and polymer chain mobility, J. Mater. Sci. 24 (1989) 1835-1844.

[12] R. Bradshaw, L.C. Brinson, Mechanical response of linear viscoelastic composite laminates incorporating non-isothermal physical aging effects, Compos. Sci. Technol. 59 (1999) 1411-1427.

[13] A. D’Amore, G. Caprino, L. Nicolais, G. Marino. Long-term behavior of PEI and PEI-based composites subjected to physical aging. Compos. Sci. Technol. 59 (1999) 1993-2003.

[14] H. Parvatareddy, P.H.W. Tsang, D.A. Dillard, Impact damage resistance and tolerance of high performance polumeric composites subjected to environmental aging, Compos. Sci. Technol. 53 (1995) 154-160.

[15] C. Wang, C.T. Sun, Experimental characterization of constitutive models for PEEK thermoplastic composite at heating stage during forming. J. Compos. Mater. 31 (1997) 1480-1506.

[16] T.Q. Li, M.Q. Zhang, K. Zhang and H.M. Zeng, The dependence of the fracture toughness of thermoplastic composite laminates on interfacial interaction. Compos. Sci. Technol. 60 (2000) 465-476.

[17] G.C. Shih, W.W. Tseng, A.Y. Lou, Evaluation of test methods in the determination of inplane shear modulus of poly(phenylene sulfide) matrix composites. Polym. Compos. 15 (1994) 1-6.

[18] C. Filiou, C. Galiotis, In situ monitoring of the fiber strain distribution in carbon fiber thermoplastic composites. 1. Application of the tensile stress field. Compos. Sci. Technol. 59 (1999) 2149-2161.

[19] L.N. McCartney, G.A. Schoeppner, W. Becker, Comparison of models for transverse ply cracks in composite laminates. Compos. Sci. Technol. 60 (2000) 2347-2359. 